\title{
An error analysis of the techniques used in the measurement of high-speed friction on snow
}

\author{
S. C. Colbeck \\ U.S. Army Cold Regions Research and Engineering Laboratory, Hanover, NH 03755-1290, U.S.A.
}

\begin{abstract}
Controlled tests are needed to find the coefficient of friction of snow as a function of speed. An error analysis shows how the test must be designed to give accurate answers. It seems necessary to use a remotely controlled, aerodynamical sled in place of a skier to get accurate results. Otherwise, two sets of tests are necessary, one to determine air drag versus speed and one to determine the frictional force versus speed, and even these tests would probably not give satisfactory results. The slope used for testing should be steep for a quick acceleration and then uniform, but not flat, where the actual measurements are taken. A continuously reading speed sensor is needed, not discrete measuring points. Even with the underlying principles understood, there will still be many practical problems to be solved before accurate results can be obtained.
\end{abstract}

\section{INTRODUGTION}

The coefficient of friction on snow is of great interest (e.g. Leino and others, 1983) but is difficult to measure even for a given set of snow and atmospheric conditions. It would be valuable to know how snow friction varies with both of those conditions, slider type and base preparation. There are many difficulties in getting measurements of the coefficient of friction in the range of speeds of most interest, i.e. greater than $5 \mathrm{~m} \mathrm{~s}^{-1}$. First, its value is always low and thus there is likely to be a large relative error. Secondly, it is necessary to separate the air and sliding resistances, which is a major problem at the speeds of interest. Tests could be done by holding a stationary slider on moving snow but not without reusing, and therefore polishing, the snow surface. At high speeds, tests need to be done outdoors, which will ensure a problem with air drag as well as some loss of control of the test conditions.

A compilation of measurements of snow friction versus speed is shown in Figure 1. With the onset of motion, friction drops rapidly below the static value because of lubrication by melting during sliding (Colbeck, in press). The friction continues to drop with increasing speed on ice surfaces, but on snow surfaces it increases after passing through a minimum. This can be explained theoretically

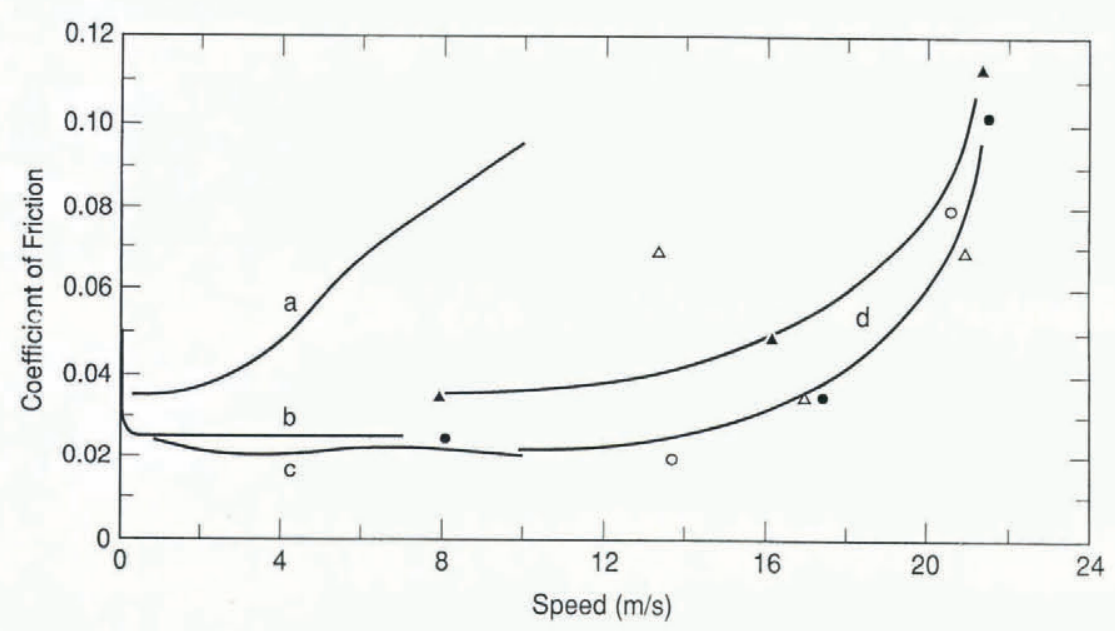

Fig. 1. Coefficient of friction versus speed as summarized by Colbeck (in press). Curve a is for dense, wet snow (from Spring, 1988). Curve $b$ is for PTFE (polytetrafluoroethylene) on wet snow (from Shimbo, 1961). Curve $c$ is for dense snow at $-7.5^{\circ} \mathrm{C}$ (from Spring, 1988). The data points (d) are for waxed (circles) and unwaxed (triangles) polyethylene on dry (solid symbols) snow at $-2.5^{\circ}$ to $-1.6^{\circ} \mathrm{C}$ and on wet (open symbols) snow (from Kuroiwa, 1977). 
by the dynamics of the meltwater films (Colbeck, 1988), but tests need to be done at the higher speeds and the results need to be established more definitely. In particular, the trends for increasing friction for wet and dry snows shown in Figure 1 are questionable because they suggest rather high values of friction at speeds that are common for both ski racing and aircraft operations.

Many attempts have been made to measure snow friction at higher speeds but there are a number of theoretical as well as practical barriers to overcome before satisfactory tests can be achieved. The theoretical limitations on friction tests are discussed here to provide a basis from which better tests can be designed. The error analysis given here is based on the concept of relative errors which has been described in Gellert and others (1989) and was used by Colbeck (1978). Relative errors are used instead of absolute errors because they show how accurate the measured or calculated quantities must be to get an accurate answer.

\section{RELATIVE ERRORS}

Assuming there is no aerodynamic lift on the slider, motion down the fall line on snow is described by

$$
m a=m g \sin \theta-f-m g \mu \cos \theta
$$

where $m$ is the mass of the slider, $a$ is its acceleration down a slope of inclination $\theta, g$ is the acceleration due to gravity, $f$ is the air drag and $\mu$ is the coefficient of friction. The first term on the righthand side represents the force due to gravity and the third term represents the frictional drag on the snow. This equation is integrated to express the conservation of energy as

$$
\frac{1}{2} m\left(v^{2}-v_{0}^{2}\right)+m g\left(y-y_{0}\right)+\int_{0}^{S}(f+m g \mu \cos \theta) \mathrm{d} S=0
$$

where $S$ is the total path length, $v$ is the speed and $y$ is the vertical coordinate. This shows that the increase in kinetic energy plus the decrease in potential energy plus the energy losses due to snow friction and air resistance must balance. While Leino and others (1983) used this energy equation to find air resistance and snow friction, Equation (1) will be used here because it is much better to measure speed continuously rather than at discrete points.

Equation (1) shows that

$$
\mu=\tan \theta-\frac{m a+f}{m g \cos \theta} .
$$

Since in most cases the first and second terms on the righthand side of this equation, or the driving and retarding terms respectively, are large but about equal in magnitude, the relative error in calculating $\mu$ from measured quantities can be quite high. Accordingly, it is necessary to design carefully a measurement program that can minimize the total error. First, it is necessary to understand the sources of error.

Assuming we know $m, g$ and $\theta$ exactly, the relative error in computing $\mu$ from measurements of speed and a calculation or measurement of air drag is

$$
E(\mu) \leq \frac{|a| E(a)}{g \mu \cos \theta}+\frac{f E(f)}{m g \mu \cos \theta}
$$

where the relative error for any variable $x$ is defined as $|\mathrm{d} x / x|$ and $a$ is $\mathrm{d} v(t) / \mathrm{d} t$, where $v(t)$ is the time series of speed. Some important conclusions are immediately obvious from this equation:

1. $\mu$ is always small and occurs in both denominators which tends to increase $E(\mu)$. Thus other factors must be optimized in order to get an acceptably small error.

2. If $E(a)$ is a constant, the measurements should be taken on a slope where the slider rapidly approaches its terminal speed so that $|a|$ is minimized. If $E(a)$ is not constant, then it is the maximum error in $|a|$ that matters and this may vary with $|a|$.

3. The common practice of calculating the speed and acceleration from timing measurements at three points is not going to give an accurate result unless $|a|$ is very small. Otherwise, averaging the speed between distinct measurement points could lead to much uncertainty. The error in this method can be reduced if the measurement points are far apart but then $a$ is likely to change significantly over the interval. Use of a rapidly reading radar gun would be much more likely to reduce $E(a)$ and then minimizing $|a|$ would not be so important. Other devices to find the time series of speed should also be considered, e.g. use of a sonic anemometer mounted on the ski or Watanabe's (1979) use of closely spaced magnetic coils.

4. $E(f)$ is more difficult to reduce because the shape of a skier is not simple and the usual formulas for calculating drag may not give satisfactory results. Nachbauer and others (1992) showed that it is necessary to improve the accuracy of the measurements and/or of the use of the formula to calculate drag. Probably the best approach is to use skis carrying a streamlined dead weight where the weight is distributed close to the ground. This could have the minimum air drag, correct weight and be stable at high speeds. Both $f$ and $E(f)$ could be minimized by using streamlined sliders rather than skiers. Since $f$ increases rapidly with increasing speed, it deserves a lot of attention even though its determination is not the primary concern. In fact, $f$ is the major problem. For a skier moving at a steady $30 \mathrm{~m} \mathrm{~s}^{-1}$, Equation (4) shows (for $\theta=0, m=80 \mathrm{~kg}$, $\mu=0.1, C_{\mathrm{D}}=0.7$ and $a=0.3 \mathrm{~m}^{2}$ ) that $E(\mu) \approx$ $1.5 E(f)$, thus $E(\mu)$ cannot be small for skier tests since $E(f)$ cannot be small.

5. $m$ could be increased to reduce the second term but weight might affect $\mu$. Thus the weight of the test system should be similar to that of the intended user of the information, e.g. a skier.

6. The effect of slope itself is minimal since only $\cos \theta$ appears in the equation. The primary effect of $\theta$ is through its influence on $v$ and $a$. If $\theta$ is not constant, use of Equations (1) and (3) could be complicated and, as shown later, errors are introduced unless the 
slope is known and uniform. Leino and others (1983) and Spring (1988) used level surfaces for their measurement zones and, while this minimizes uncertainty in $\theta$ and maximizes $\cos \theta$, minimizing $|a|$ may be more important, especially if discrete measurement points are used to get $v$ and $a$.

7. The ratio $f / m$ can be minimized by using a heavy, flat plate mounted horizontally on two skis. The drag would be all surface drag and would typically be reduced by a factor of 100 over a streamlined skier, but use of such a plate may be impractical because of the lack of control.

Of the two terms in Equation (4), the effect of air drag is the most uncertain and thus it deserves special consideration. The slope of the test track is discussed later because it should be chosen to minimize both terms.

\section{AIR DRAG}

The air drag on a three-dimensional object is given by

$$
f=0.5 \rho v^{2} C_{\mathrm{D}} A
$$

where $C_{\mathrm{D}}$ is the coefficient for form drag and $A$ is the reference area for the body. Reynolds number is given by

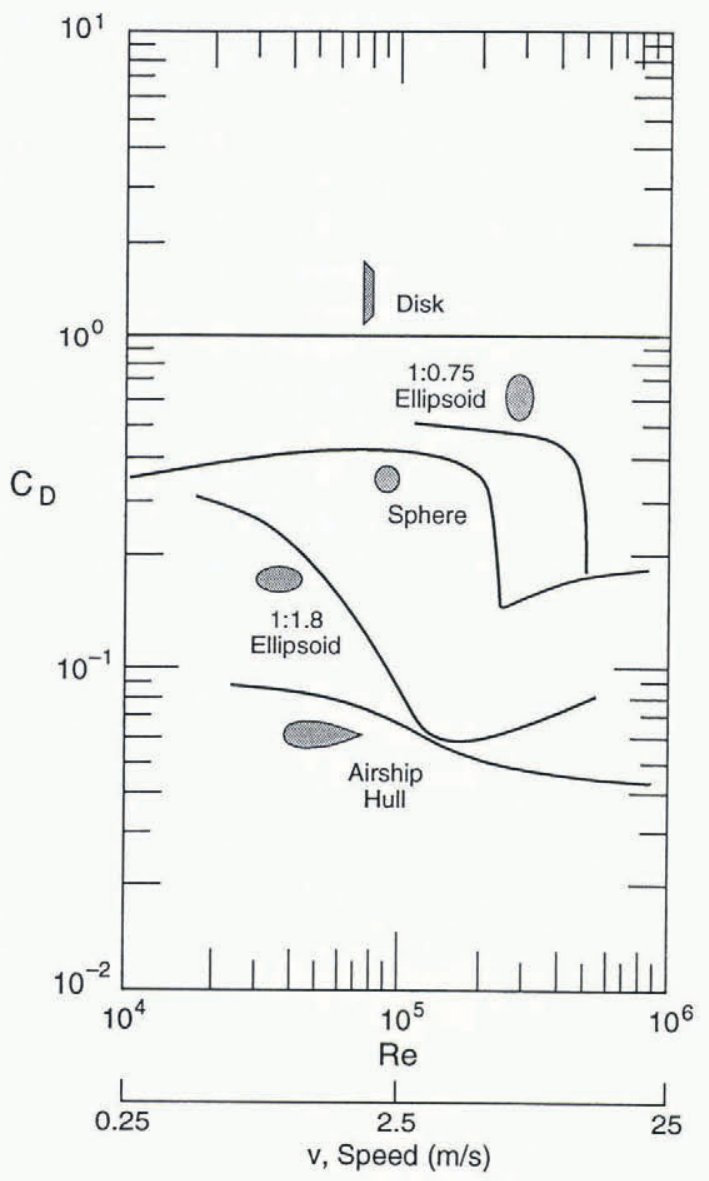

Fig. 2. Drag coefficient versus Reynolds number and speed for various bodies (data from Rouse and Howe, 1953). The speed is calculated assuming $\ell$ of $0.5 \mathrm{~m}$ and $\nu$ of $1.25 \times 10^{-5} \mathrm{~m}^{2} \mathrm{~s}^{-1}$.

$$
\operatorname{Re}=\frac{v \ell}{\nu}
$$

where $\ell$ is a characteristic dimension and $\nu$ is the kinematic viscosity of air, and it is used to characterize the mode of flow. For values of Re greater than about $10^{5}$, which are of primary interest here, Figure 2 shows that there is a sudden drop in $C_{\mathrm{D}}$ for ellipsoids and spheres. This occurs because the boundary layer changes suddenly to turbulent flow as the air speed increases in this range. This is a severe and common problem in tests involving air drag (Hoerner, 1965). Furthermore, the tests may not be repeatable since separation often occurs at different positions along a body in different tests, even at the same speed.

A skier has little control over Re although $\ell$ can be changed slightly, but it is important to recognize that air drag increases with $\operatorname{Re}$ after $C_{\mathrm{D}}$ goes through a minimum and that, for a given $C_{\mathrm{D}}, f$ increases as $v^{2}$. Thus, above a certain speed, air drag increases rapidly due to increases in both $v$ and $C_{\mathrm{D}}$. This is a critical point because $f$, and therefore $E(\mu)$, can be decreased by designing an experiment that will take advantage of the minimum in the relationship between $C_{\mathrm{D}}$ and $\mathrm{Re}$. For example, for the $1: 1.8$ ellipsoid oriented with the flow, the tests should be done in the range of $10^{5} \leq \mathrm{Re} \leq 10^{6}$, which corresponds to a sliding speed of about $2.5-25 \mathrm{~m} \mathrm{~s}^{-1}$. By coincidence, this is a range of great interest.

Two approaches for finding air drag on a threedimensional object can be taken. The best approach is to find $f$ as a function of $v$ by passing the object through still air and measuring $f$ and $v$ very accurately. Then, given a high accuracy in the resolution of $f$ versus $v, E(\mu)$ would be relatively little affected by the second term in Equation (4). The results would have to be corrected for the values of air density $(\rho)$ at each test site, but this can be done with simple measurements of barometric pressure, $p$, and temperature, $T$, using the formula

$$
\rho=\rho_{0} \frac{p}{p_{0}} \frac{T_{0}}{T},
$$

where $\rho_{0}, p_{0}$ and $T_{0}$ are at a standard reference point. $\rho$ can also be corrected for humidity, but this correction is small and can probably be ignored.

The second approach is to use Equation (5) directly and eliminate the need for the second set of tests. While this approach would be very convenient, it could introduce a great deal of error because of uncertainty in both $C_{\mathrm{D}}$ and $A$. The relative error in $f$ is given by

$$
E(f) \leq E(\rho)+2 E(v)+E\left(C_{\mathrm{D}}\right)+E(A) .
$$

Since $p$ and $T$ can be measured precisely and the change in $\rho$ due to humidity is small, $E(\rho)$ can probably be ignored. If $v$ can be resolved accurately, the second term should be negligible, but the factor of 2 is important to consider. The uncertainty in both $C_{\mathrm{D}}$ and $A$ could be reduced with an aerodynamical dead weight but, because of the variation of $C_{\mathrm{D}}$ with speed over the range of interest, it is not clear that $E(f)$ can be kept within 
acceptable bounds without a separate set of measurements. Unfortunately, these tests in themselves will be difficult, especially if skiers are used. Spring (1988) showed that air-drag measurements of skiers were subject to very large uncertainties at speeds of less than $5 \mathrm{~m} \mathrm{~s}^{-1}$ and there was still a large relative error above that speed.

Of the shapes shown in Figure 2, the one that is least affected by speed is the disk, probably because separation occurs at the edge at any speed. Thus, the disk is an attractive shape because its drag can be readily calculated with little error and good repeatability. In addition, $f$ and terminal speed could be varied independently of $m$ by changing the diameter. However, $E(\mu)$ increases as $f E(f)$ and $f$ for a disk is about 50 times greater than for an airship hull.

Use of a disk would also avoid the effects of surface roughness. Surface-roughness elements are critical, for example, in the flight of a golf ball (Mehta, 1985). However, we assume here that the surfaces are smooth or that the same object is used for both the air-drag and snow-slope tests.

\section{SLOPE ANGLE}

Expressing acceleration as $\mathrm{d} v / \mathrm{d} t$ in Equation (1) and combining with Equation (5),

$$
m \frac{\mathrm{d} v}{\mathrm{~d} t}=m g \sin \theta-0.5 \rho v^{2} C_{\mathrm{D}} A-m g \mu \cos \theta .
$$

To discuss the effects of slope angle, we will find $v$ versus $t$ by ignoring any dependence of $C_{\mathrm{D}}$ on $\mathrm{Re}$ and assuming that $\theta$ does not vary along the track of known slope. For a streamlined dead weight in a properly designed test, the first assumption would be reasonable if speed increased quickly into the range where $C_{\mathrm{D}}$ is fairly constant.

Integrating Equation (9) from an initial speed $v_{0}$ yields

$$
\frac{v_{\infty}+v}{v_{\infty}-v}=\frac{v_{\infty}+v_{0}}{v_{\infty}-v_{0}} \exp \left(\rho C_{\mathrm{D}} A v_{\infty} t / m\right)
$$

where the terminal speed is given by

$$
v_{\infty}^{2}=\frac{2 m g(\sin \theta-\mu \cos \theta)}{\rho C_{\mathrm{D}} A} .
$$

For a slope of $10^{\circ}$, a slider of $90 \mathrm{~kg}, \mu$ of $0.1, C_{\mathrm{D}}$ of 0.5 and $A$ of $0.25 \mathrm{~m}^{2}$, the terminal speed would be about $28 \mathrm{~m} \mathrm{~s}^{-1}$. This speed seems high for these values of $C_{\mathrm{D}}$ and $\theta$ which suggests that $\mu$ may indeed increase with speed, as suggested in Figure 1.

For a constant slope, Figure 3 shows that the slider would change speed very rapidly at first. However, because $f$ increases as $v^{2}$, the terminal speed is approached slowly. There is a slight advantage in overshooting the terminal speed before entering the test part of the slope because the slider would approach $v_{\infty}$ more rapidly while decelerating than while accelerating.

The slope of the test track should be chosen to minimize the first term in Equation (4), in particular $|a| E(a) / \cos \theta$. From Equation (1), this requires a

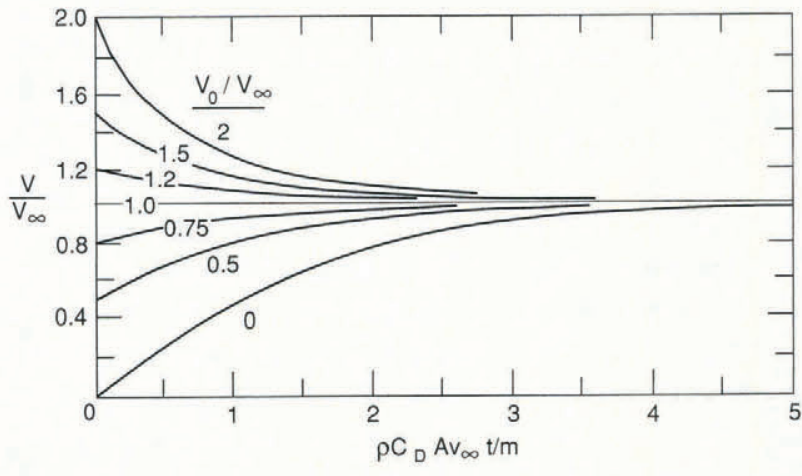

Fig. 3. Speed versus time calculated from Equation (10) and given in dimensionless form with initial over final speed as a parameter.

minimum of

$$
\left|g \tan \theta-\frac{f}{m \cos \theta}-g \mu\right| E(a) .
$$

While the first two terms can be minimized by making the measurements on a level section of track, the entire expression can be minimized by choosing a sloping section of track of sufficient length to allow the slider to approach its terminal speed, thus minimizing $|a|$. For typical conditions from a standing start on a track of constant slope, this would require about $1 \mathrm{~min}$ of time, which is much too great. Thus, the advantage of using a concave track, as suggested by Leino and others (1983), with a length of constant slope near the bottom is obvious. Unfortunately, as shown in Figure 3, even this approach will not allow a quick attainment of the terminal speed unless it is achieved before the slider reaches the test section. Thus, while it is desirable to make the tests at a constant speed, it may be impractical to do so. Furthermore, the result would only produce one value for $v_{\infty}$ on each slope for each set of conditions, but at least this approach offers the possibility of accurate tests that could be used to determine the effects of different ski-base preparations or snow conditions. The inclination of the steeper part is not critical since only a part of that slope

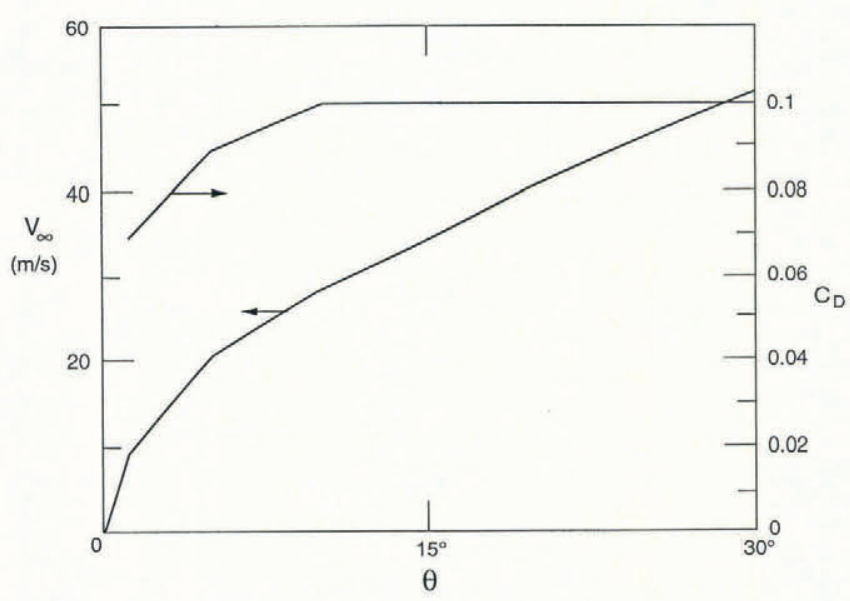

Fig. 4. Terminal speed and drag coefficient versus slope of the run for a $1: 1.8$ ellipsoid oriented with the flow. 
would need to be used to achieve the desired speed. The critical part of the slope is the lower part where the measurements are to be made at minimum $|a|$.

$v$ was calculated as a function of $\theta$ for a $1: 1.8$ ellipsoid oriented with the flow using Equation (11) and Figure 2 as guidance to find $C_{\mathrm{D}}$. At each value of $\theta, C_{\mathrm{D}}$ was determined by iterating to the solution while correcting $C_{\mathrm{D}}$ at each step. The result is shown in Figure 4, where it can be seen that $v_{\infty}$ increases with slope at a significant rate through the range of interest. From Equation (3), when all other errors are zero, the error in $\mu$ due to an error or perturbation in the gradient of the slope is given by

$$
E(\mu)=\frac{1+\mu \tan \theta}{\mu} \theta E(\theta) .
$$

Since $1 \gg \mu \tan \theta$, this can be approximated as

$$
E(\mu) \approx \frac{\theta}{\mu} E(\theta)
$$

which, for slopes greater than a few degrees, shows that $E(\mu)$ will be greater than $E(\theta)$. Thus, where the actual measurements are taken, the slope must be very uniform to minimize $E(\mu)$.

\section{DESIGN OF THE SLIDER}

Assuming the slider will consist of two runners loaded with a weight, it is then necessary both to minimize the air drag and to know precisely how it varies with speed. The second term in Equation (4) shows that $E(\mu)$ increases as $f E(f)$, or $E(\mu)$ increases with the uncertainty in $f$. This can be minimized by reducing both $f$ and the uncertainty in determining $f$ from tests. While a skier provides the best control of the skis, use of a skier presents two major problems, First, the skier's frontal area and drag coefficient are high. Secondly, there is likely to be poor reproducibility in either transferring wind-tunnel results to ski slopes or in repeated tests on a given slope. This is shown in Spring's (1988) figure 2, where, even at the higher speeds, there is much scatter in the measured results, possibly due to slight changes in the skier's configuration.

Use of a rigid, streamlined sled would allow reduction of $C_{\mathrm{D}}$, increase confidence in transferring results from air-drag tests to ski slopes and improve repeatability among tests. Wind-tunnel results show rather little experimental uncertainty when using streamlined objects (Rouse and Howe, 1953; Hoerner, 1965), and these results could be improved by passing the object through still air. Use of an object of the shape of an airship hull would greatly reduce $C_{\mathrm{D}}$ and provide housing for steering and braking mechanisms and weights. This shape also offers the advantage that, by adjusting its aspect ratio, it may be possible to minimize drag in a range of values of $\mathrm{Re}$ where there is little variation in $C_{\mathrm{D}} \cdot C_{\mathrm{D}}$ decreases as the aspect ratio of rotationally symmetric bodies increases. Thus, elongation of the airship hull decreases $C_{\mathrm{D}}$ while increasing the value of Re at which the minimum $C_{\mathrm{D}}$ occurs (Hoerner, 1965). For example, using a rotationally symmetric body with an aspect ratio of 8 and a length of $2 \mathrm{~m}$ would give a value of $C_{\mathrm{D}}$ of about 0.0025 (Hoerner, 1965), which would be fairly constant over the range of speeds of interest $(0.002$ 0.003 for any speed greater than $3 \mathrm{~m} \mathrm{~s}^{-1}$ ). From the second term in Equation $(4), E(\mu) \approx 0.00085 E(f)$ at a constant speed of $30 \mathrm{~m} \mathrm{~s}^{-1}, \mu=0.1, m=80 \mathrm{~kg}$ and $\theta=0$. Thus the error due to air drag would be essentially eliminated and it seems likely that a test could be done on a slope of constant angle with little effect of air drag. It should even be acceptable to bypass the wind-tunnel tests. However, use of a steering fin would increase the drag and reduce the repeatability among the tests.

\section{SUMMARY}

Various attempts have been made to measure the coefficient of friction for skis on snow but the results are not sufficiently accurate. In fact, use of a skier probably precludes accurate results since the air drag is too large and the measurements are not repeatable. It is necessary to use a sled shaped like an airship hull with an aspect ratio of about 8 . This would minimize the drag, increase repeatability and, for speeds greater than $3 \mathrm{~ms}^{-1}$, minimize the change in $C_{\mathrm{D}}$ due to the transition from laminar to turbulent flow. Unlike for the human form, the variation in $C_{\mathrm{D}}$ would be small enough and the drag low enough to calculate the drag and bypass wind-tunnel tests. For a slope of at least $10^{\circ}$, the uncertainty in the air drag would be small enough to ignore.

Use of a continuously reading speed sensor is necessary unless the slider approaches its terminal speed. This can best be achieved by over-accelerating on a steep slope and then running at the terminal speed on a constant, but not level, slope that gives the desired speed.

There are other problems to be solved besides those discussed here. The design and construction of an unmanned sled presents engineering challenges and its use on many slopes will be tricky. Since the surface conditions of the snow greatly affect the friction, the surface conditions must be controlled or chosen carefully to ensure that tests are representative of the desired conditions. It may be difficult to find ski slopes which have the ideal shape for quick acceleration followed by a constant speed. While these practical considerations remain to be solved by experimentalists, the guiding principles outlined here must be considered to optimize the chances of achieving reasonable results.

\section{ACKNOWLEDGEMENTS}

I thank George Ashton, Bert Davis and a reviewer for their comments on this manuscript. This work was motivated by discussions with Michel Tavernier of Fédération Française de Ski. The work was supported at CRREL by Physical Properties of Snow Covers.

\section{REFERENCES}

Colbeck, S. C. 1978. The difficulties of measuring the water saturation and porosity of snow. F. Glaciol., 20 (82), 189201. 
Colbeck, S. C. 1988. The kinetic friction of snow. J. Glaciol., 34(116), 7886.

Colbeck, S. C. In press. A review of the friction of snow ski. 7. Sport Sci.

Gellert, W., S. Gottwald, H. Hellwich and H. Kustner, eds. 1989. The VNR concise encyclopedia of mathematics. Second edition. New York, Van Nostrand Reinhold.

Hoerner, S. F. 1965. Fluid-dynamic drag. Midland Park, NJ, Hoerner.

Kuroiwa, D. 1977. The kinetic friction on snow and ice. $\mathcal{J}$. Glaciol., 19(81), 141-152.

Leino, M.A.H., E. Spring and H. Suominen. 1983. Methods for the simultaneous determination of air resistance to a skier and the coefficient of friction of his skis on snow. Wear, 86, 101-104.

Mehta, R. D. 1985. Aerodynamics of sports balls. Annu. Rev. Fluid Mech., 17, 151-189.

Nachbauer, W., P. Kaps and M. Mossner. 1992. Determination of kinetic friction in downhill skiing. In VIII Meeting of European Society of Biomechanics, Rome, 21-24 June 1992.
Rouse, H. and J. W. Howe. 1953. Basic mechanics of fluids. New York, John Wiley.

Shimbo, M. 1961. The mechanism of sliding on snow. International Association of Scientific Hydrology Publication 54 (General Assembly of Helsinki 1960 - Snow and Ice), 101-106.

Spring, E. 1988. A method for testing the gliding quality of skis. Tribologia, 7(1), 9-14.

Watanabe, K. 1979. Measurement of the speed of skiing throughout the turn by the "coil magnet system". In Terauds, J. and J. J. Gros, eds. Science in skiing, skating and hockey. Del Mar, CA, Academic Publishers, $41-48$.

The accuracy of references in the text and in this list is the responsibility of the author, to whom queries should be addressed. 